\title{
Determining Prosthesis-Patient Mismatch after TAVR: Which is the Best Method?
}

\author{
Cesar Mendoza ${ }^{1}$ and Diego Celli ${ }^{2}$ \\ ${ }^{1}$ university of miami \\ ${ }^{2}$ University of Miami Health System
}

October 12, 2020

\section{Determining Prosthesis-Patient Mismatch after TAVR: Which is the Best Method?}

Authors: Cesar E. Mendoza, $\mathrm{MD}^{1}$ and Diego Celli, $\mathrm{MD}^{2}$

Affiliations: ${ }^{1}$ Division of Cardiovascular Disease, Jackson Memorial Hospital, Miami, Florida; ${ }^{2}$ Internal Medicine, University of Miami Miller School of Medicine/Jackson Memorial Hospital, Miami, Florida.

Affiliation addresses: ${ }^{1} 1801 \mathrm{NW} 9{ }^{\text {th }}$ Ave, Suite \#209 33136 Miami, Florida, United States; ${ }^{2} 1611$ NW 12th Ave 33136, Miami, Florida, United States.

Corresponding author: Cesar E. Mendoza, MD; cesar.mendoza2@jhsmiami.org; $1611 \mathrm{NW} 12^{\text {th }}$ Ave, East Tower 3019, Miami, Florida 33136.

Disclosures: Authors have no relationships with the industry. This work is not under consideration in any other journal.

Funding: No grants, contracts, and other forms of financial support were used to perform this manuscript.

In the last decade, the medical community has witnessed an accelerated development of multiple devices for the transcatheter management of aortic stenosis. Recently, transaortic valve replacement (TAVR) was granted approval for its use in all types of surgical risk patients underscoring its importance in cardiovascular practice. While evidence has shown non-inferiority of TAVR versus surgical aortic valve replacement (SAVR) [1], it still has inherent intra- and post-procedural complications, prosthesis-patient mismatch (PPM) is one of them.

Since the seminal work published by Rahimtoola in 1978 [2], several studies have investigated PPM. The incidence of PPM after SAVR ranges from $20 \%$ to $50 \%$ with severe cases having an occurrence rate from $5 \%$ to 25\%. [3-5]. Severe PPM has been associated with significantly abnormal prosthetic valve echocardiographic parameters and adverse clinical outcomes including a higher risk of mortality [3,5-7]. Although initial studies showed a lower incidence of PPM after TAVR [8, 9], most recent data surprisingly depict an uptrend incidence of PPM with later-generation TAVR prostheses [10]. Regardless of the true global PPM incidence, the number of cases in the severe category remain within robust margins $(5 \%-36 \%)$. Perhaps, more interestingly, the association of TAVR with adverse outcomes is not firm. Indeed, there are conflicting reports, with some studies showing a weak association [11,12], no association $[13,14,15]$, or association in particular group of patients [9].

PPM occurs when the effective orifice area (EOA) of a normally functioning prosthesis is too small in relation to the patient's body size and cardiac output requirements, and this diagnosis must be done after ruling out dysfunction of the prosthesis heart valve. Historically, surgical aortic valve replacement was the method of choice in the management of aortic stenosis; as such, surgeons relied on the manufacturer's predicted EOA 
charts to aid in the determination of the minimum valve size for any given valve model. The predicted EOA index (EOAi), which is calculated by dividing the reference value for the prosthesis model and size by the body surface area (BSA) of the patient, has been frequently used to identify PPM in the SAVR studies. Similarly, all contemporary TAVR studies have used the same index for the same purpose; but it nevertheless was measured using Doppler-echocardiography data.

In this issue of JOCS, Catalano et al report that the utility of EOAi charts to predict PPM after TAVR for native aortic stenosis may be limited. Indeed, they found in their study that the pre-TAVR prediction of PPM using tables of expected EOA varies significantly from actual PPM measured on intraoperative transesophageal echocardiography using the continuity equation. Although this is a relatively small singlecenter study, the authors provided information worthy of additional consideration.

First, they identified that EOAi charts overestimated the number of patients with PPM for Sapien 3 valves ( $25.3 \%$ predicted versus $13.7 \%$ actual) and underestimated the number of patients with PPM for Evolut valves (1.8\% predicted versus $11.6 \%$ actual), yielding a limited utility for this instrument on pre-operative prediction of PPM in TAVR. Interestingly, a recent publication by Ternacle et al. [16] provides a different perspective on this topic. It reports that the predicted EOAi was found to be useful to reclassify the majority of patients diagnosed with measured PPM following TAVR to no PPM at all. Furthermore, they found that both methods had a different association with hemodynamic outcomes. In this regard, EOAi and mean transprosthetic gradient had a more powerful correlation when using the predicted EOAi versus the measured EOAi. Based on these findings, the Ternacle's study suggests that the use of measured EOAi grossly overestimates the incidence of PPM. The discrepancy between both studies may be explained by the inherent variability in using different Doppler echocardiography imaging modalities to measure EOA. As Catalano et al rightly pointed out, the prosthesis data acquisition and measurements obtained by intraoperative transesophageal echocardiography in their study may not be comparable with its counterpart transthoracic modality, and this particular difference should be taken into account when interpreting the results above mentioned.

Second, it is also clear from Catalano's study that determining the best method to diagnose PPM following TAVR is paramount, but at the same time troublesome due to several factors. First, the pressure recovery phenomenon, a portion of the transprosthetic pressure gradient lost initially at the vena contracta level that recovers later after the prosthetic valve, is not accounted for by Doppler assessment of the maximum transvalvular flow velocities. This may cause overdiagnosis of PPM after TAVR. Second, measured EOA is influenced by the patient's hemodynamic condition at the time of the evaluation and by the known technical pitfalls on the acquisition of images and measurement performance. Third, the use of the EOA indexed for body surface area may overestimate the severity of PPM in obese patients (body mass index [?]30 kg/m2).

Certainly, Catalano's study allows for a better discussion on the diagnosis and clinical implications of PPM following TAVR. However, the question of what method is a more accurate parameter to determine PPM remains unanswered. Clearly, further research is needed as TAVR is more frequently performed and new TAVR prostheses become available. Accurate prediction of PPM in this setting will help guide the operator's decision on proper prosthesis size and type.

\section{References}

1. Voigtländer L, Seiffert M. Expanding TAVI to Low and Intermediate Risk Patients. Front Cardiovasc Med. 2018;5:7-12.

2. Rahimtoola SH. The problem of valve prosthesis mismatch. Circulation 1978; 58:20-4.

3. Dayan V Vignolo G Soca G Paganini JJ Brusich D Pibarot P. Predictors and outcomes of prosthesis patient mismatch after aortic valve replacement. JACC Cardiovasc Imaging. 2016;9:924-33.

4. Fallon JM DeSimone JP Brennan JM O'Brien S Thibault DP DiScipio AW et al. The incidence and consequence of prosthesis-patient mismatch after surgical aortic valve replacement. Ann Thorac Surg 2018;106:1422 . 
5. Pibarot P Clavel MA. Prosthesis-patient mismatch after transcatheter aortic valve replacement: it is neither rare nor benign. J Am Coll Cardiol 2018;72:2712-6.

6. Head S Mokhles M Osnabrugge R Pibarot P Mack MJ Takkenberg J et al. The impact of prosthesis-patient mismatch on long-term survival after aortic valve replacement: a systematic review and meta-analysis of 34 observational studies comprising 27,186 patients with 133,141 patient-years. Eur Heart J 2012;33:1518-29.

7. Sa M de Carvalho MMB Sobral Filho DC Cavalcanti LRP Rayol SDC Diniz RGS et al. Surgical aortic valve replacement and patient-prosthesis mismatch: a meta-analysis of 108182 patients. Eur J Cardiothorac Surg 2019;56:44-54.

8. Thyregod HG Steinbruchel DA Ihlemann N Ngo TA Nissen H Kjeldsen BJ et al. No clinical effect of prosthesis-patient mismatch after transcatheter versus surgical aortic valve replacement in intermediateand low-risk patients with severe aortic valve stenosis at mid- term follow-up: an analysis from the NOTION trial. Eur J Cardiothorac Surg 2016;50:721-8.

9. Pibarot P Weissman NJ Stewart WJ Hahn RT Lindman BR McAndrew T et al. Incidence and sequelae of prosthesis-patient mismatch in transcatheter versus surgical valve replacement in high-risk patients with severe aortic stenosis- A PARTNER trial cohort A analysis. J Am Coll Cardiol 2014;64:1323-34.

10. Pibarot P. The CHOICE between self-expanding and balloon expandable valves for transcatheter aortic valve replacement. J Am Coll Cardiol Intv 2018; 11:2519-22

11. Herrmann HC Daneshvar SA Fonarow GC Stebbins A Vemulapalli S Desai ND et al. Prosthesis-patient mismatch in patients undergoing transcatheter aortic valve replacement: from the STS/ACC TVT registry. J Am Coll Cardiol 2018;72:2701-11.

12. Ewe SH Muratori M Delgado V Pepi M Tamborini G Fusini L et al. Hemodynamic and clinical impact of prosthesis-patient mismatch after transcatheter aortic valve implantation. J Am Coll Cardiol 2011;58:1910-8.

13. Thyregod HG Steinbruchel DA Ihlemann N Ngo TA Nissen H Kjeldsen BJ et al. No clinical effect of prosthesis-patient mismatch after transcatheter versus surgical aortic valve replacement in intermediate- and low-risk patients with severe aortic valve stenosis at mid-term follow-up: an analysis from the NOTION trial. Eur J Cardiothorac Surg 2016;50:721-8.

14. Tzikas A Piazza N Geleijnse ML Van Mieghem N Nuis RJ Schultz C et al. Prosthesis-patient mismatch after transcatheter aortic valve implantation with the medtronic CoreValve system in patients with aortic stenosis. Am J Cardiol 2010;106:255-60.

15. Miyasaka M Tada N Taguri M Kato S Enta Y Otomo T et al. Incidence, predictors, and clinical impact of prosthesis-patient mismatch following transcatheter aortic valve replacement in Asian Patients: the OCEANTAVI Registry. JACC Cardiovasc Interv 2018;11:771-80.

16. Ternacle J, Guimaraes L, Vincent F, et al. Reclassification of prosthesis-patient mismatch after transcatheter aortic valve replacement using predicted vs. measured indexed effective orifice area.European Heart Journal Cardiovascular Imaging . Published online September 30, 2020. doi:10.1093/ehjci/jeaa235 